\title{
Effects of Self-Awareness of Eating Behaviors and Differences in Daily Habits Among Japanese University Students on Changes in Weight and Metabolism
}

\author{
Tetsuya Kakuma ${ }^{\mathrm{a}, \mathrm{d}}$, Yuichi Yoshida ${ }^{\mathrm{b}}$, Mitsuhiro Okamoto $^{\mathrm{b}}$, Hirotaka Shibata ${ }^{\mathrm{b}}$, \\ Takashi Tsutsumic, Yoshikuni Kudo ${ }^{\mathrm{c}}$
}

\begin{abstract}
Background: In addition to daily weight measurements and regular exercise, not skipping breakfast, refraining from eating at night, and not overconsuming soft drinks have been reported to suppress the onset and progression of obesity and metabolic syndrome in adulthood. However, few studies have examined the associations between these daily lifestyle habits and the types of eating behaviors (e.g., food preferences, conception of eating, eating habits) among university students.
\end{abstract}

Methods: We investigated the characteristics of eating behaviors based on backgrounds and lifestyle factors in association with changes in weight and metabolism using blood sampling data, a questionnaire on eating behaviors conducted during clinical training, and data from regular health examinations of 100 fifth-grade students at the Oita University Faculty of Medicine in Japan.

Results: Characteristic eating behaviors, including daily self-weighing, regular exercise, skipping breakfast, frequently eating late at night, and excess soft drink consumption, were observed for each lifestyle. In addition, three eating behaviors (fast eating, eating late-night snacks, and not eating breakfast) were extracted as factors that cause weight gain of $3 \%$ or more from the weight at the time of admission to university. Self-awareness of fast eating was significantly associated with higher body mass index in the fifth grade $(\mathrm{P}<0.001)$, and systolic blood pressure and fasting plasma glucose tended to be higher in students who were strongly aware that they would not have breakfast ( $\mathrm{P}=0.071$ and $\mathrm{P}=0.053$, respectively).

Manuscript submitted August 24, 2020, accepted September 19, 2020

Published online October 16, 2020

aHealth Support Center, Hasama Campus, Oita University, Yufu City, Oita 879-5593, Japan

bDepartment of Endocrinology, Metabolism, Rheumatology and Nephrology, Faculty of Medicine, Oita University, Yufu City, Oita 879-5593, Japan

'Health Support Center, Dannoharu Campus, Oita University, Oita City, Oita 870-1192, Japan

${ }^{\mathrm{d}}$ Corresponding Author: Tetsuya Kakuma, Health Support Center, Hasama Campus, Oita University, 1-1 Idaigaoka, Hasama-machi, Yufu City, Oita 8795593, Japan.Email: kakuma@oita-u.ac.jp

doi: https://doi.org/10.14740/jem687
Conclusions: The results indicated that the habits of "fast eating" and "not eating breakfast" respectively increase weight and may cause metabolic disorders, regardless of current weight. Thus, it is important for students to be self-aware of unhealthy eating behaviors in daily life. Although it was developed for the medical treatment of obese patients, the questionnaire on eating behaviors may be useful for helping university students learn eating behavior habits and peculiarities as well as health education.

Keywords: Fast eating; Skipping breakfast; Late-night snacking; College student; Questionnaire on eating behaviors; Weight gain; Metabolic change

\section{Introduction}

It has been noted that about $70-80 \%$ of the cases of adolescent obesity shift to adult obesity [1]; therefore, health education for university students transitioning from adolescence to adulthood could be extremely important for preventing the development and progression of adult obesity and metabolic syndrome. However, the types of lifestyles led by students that lead to obesity remain unclear. After students enter university, their lifestyles tend to change substantially (e.g., living alone, having a part-time job, attending drinking parties), and some studies have reported that the living environment itself contributes to weight gain $[2,3]$. In addition to a lack of exercise, some lifestyle patterns, such as skipping breakfast, nocturnal life, and the overconsumption of soft drinks, have been reported to be associated with adolescent weight gain [3-7]. However, even if an unfavorable lifestyle is observed, it is often the case that obesity does not necessarily occur, and metabolic abnormalities are not apparent because this generation has less visceral fat accumulation and good insulin sensitivity [8]. Therefore, to gain a better understanding of the changes in weight and metabolism among this generation, the interrelationship between lifestyle and eating habits needs to be clarified. However, to our knowledge, few studies have examined in detail what kinds of eating behaviors university students with such daily lifestyles have (e.g., whether students who skip breakfast consume more late-night snacks). Furthermore, most previous reports related to lifestyle patterns or eating habits 
have used dichotomous choice methods (i.e., "yes" or "no"), and no studies have investigated the strengths and weaknesses of habituality by specifically comparing eating habits with lifestyle patterns.

Therefore, in this study, we used a questionnaire on eating behaviors developed by Dr. Toshiie Sakata, a former professor in our department, to assess the eating behaviors of obese patients. This questionnaire was designed based on the language actually spoken by patients who had visited an outpatient clinic specializing in obesity treatment and been hospitalized for treatment according to medical records. This questionnaire has been described in the Obesity Practice Guidelines 2016 and is one of the behavioral therapies officially recognized by the Japan Society for the Study of Obesity [9]. Unlike other questionnaires that focus on food intake details (e.g., the food frequency questionnaire [10]), the present measure specifies the food preferences, conception of eating, and eating habits of obese patients, but it is not suitable for calculating caloric intake. However, based on the structure of the questionnaire and the response items, it is easy to recognize the characteristics of respondents' eating behaviors (e.g., their vulnerability to obesity). That is, the closer a response to four points in the questionnaire is, the more that eating behavior can then be interpreted as "habitual".

Given this background, in this study, we examined the characteristics of eating behavior based on the backgrounds and lifestyles of fifth-year medical students and the factors related to weight gain using blood sampling data, a questionnaire survey conducted during clinical training, and data from regular health examinations in Japan.

\section{Patients and Methods}

\section{Participants}

The participants were 100 fifth-grade medical students (63 men and 37 women) who participated in clinical training at the Department of Endocrinology, Metabolism, Rheumatology and Nephrology at the Oita University Faculty of Medicine in 2015. A retrospective analysis was performed using fasting blood sampling data, a lifestyle-focused questionnaire, a questionnaire on eating behaviors, physical characteristics (e.g., height, body weight), and blood pressure data from regular health examinations carried out in the spring. Informed consent for obtaining blood sampling data, information on physical characteristics, and questionnaire responses were obtained from all students while in school. This study was conducted in accordance with the Declaration of Helsinki and carried out with the approval of the Ethics Committee of Oita University (date of approval: June 22, 2020; approval No.: 1873).

In brief, the clinical data and questionnaire responses for eating behavior were compared based on the presence or absence of representative lifestyles, and the relationship with each lifestyle was examined. In addition, we divided the students into three groups according to weight change after university admission, compared their clinical data and questionnaire responses in regard to eating behaviors, and then ex- tracted characteristic eating behaviors related to weight gain. Finally, we examined the students' characteristic eating behaviors and trends in metabolic parameters.

\section{Laboratory measurements}

We used fasting blood sampling data obtained from the same students for a study on the effects of low-carbohydrate bread on glucose and lipid metabolism (date of approval: May 21, 2015; approval No.: 837). Plasma glucose and insulin levels were measured using standard laboratory procedures at the Oita University Hospital clinical laboratory.

\section{Lifestyle-focused questionnaire and questionnaire on eat- ing behaviors}

Questionnaires focused on lifestyle and eating behaviors were administered to all participants the day before blood collection. The lifestyle questionnaire is a "yes/no" questionnaire that has been used in previous reports $[8,11]$. Daily self-weighing was classified as "yes" (including "morning once a day", "evening once a day", and "more than once a day") or "no" (none). The rare cases of self-weighing less than once a day, such as weekly or monthly, were combined into the "none" category. Regular exercise was classified as "yes" (member of an athletic club or exercising more than 3 days a week) or "no". Skipping breakfast was classified as "yes" (no breakfast more than 3 days a week) or "no". Frequently eating late at night was classified as "yes" (eating after 22:00 more than 3 days a week) or "no". Excessive soft drink consumption was classified as "yes" (more than $500 \mathrm{~mL}$ of soft drink intake more than 3 days a week) or "no".

The questionnaire on eating behaviors has been officially approved by the Japan Society for the Study of Obesity [9] and consists of 55 question items regarding recognition of weight and constitution, external eating behavior, emotional eating behavior, sense of hunger or satiety, eating style, food preferences, and regularity of eating habits. The respondents answer the statements on a four-point scale (1. Never, 2. Sometimes, 3 . Frequently, and 4. Always), with a high score indicating a habitual eating behavior.

\section{Data from health examinations}

Gender was self-reported, and age, height, body weight, systolic blood pressure (SBP), and diastolic blood pressure (DBP) were obtained through routine physical exams conducted in the spring. Based on the height and weight values, body mass index (BMI) was calculated as the weight in kilograms divided by the height in meters squared. The changes in weight and age after admission to university were calculated as the follows: $\Delta$ age $=$ age in fifth grade (years) - age in first grade (years), and $\Delta$ weight $=$ weight in fifth grade $(\mathrm{kg})$ - weight in first grade $(\mathrm{kg})$. BMI $\geq 25 \mathrm{~kg} / \mathrm{m}^{2}$ was considered to indicate obesity [12]. 
Table 1. Clinical Characteristics and Lifestyle Profiles of the Study Participants

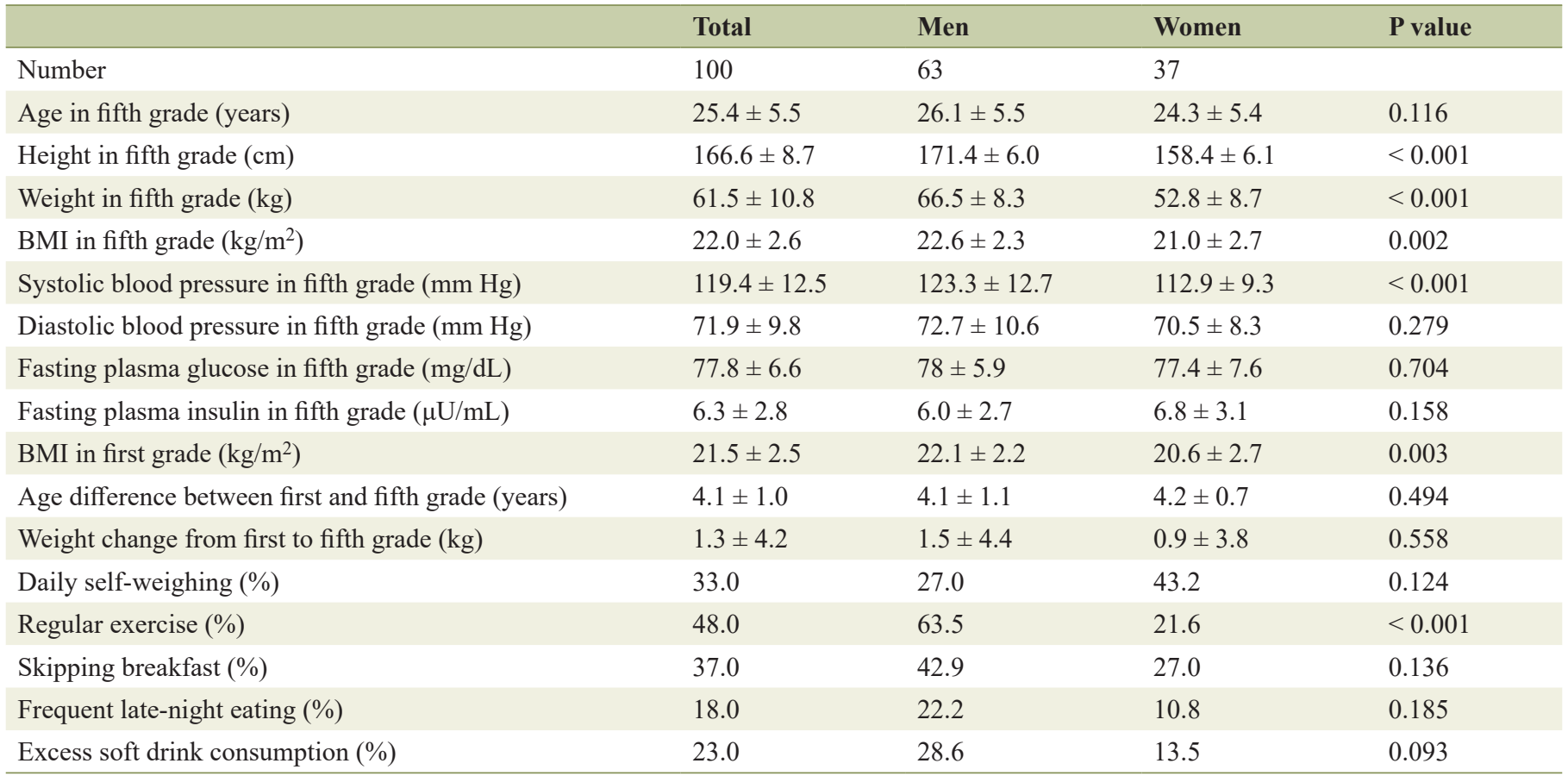

Data are expressed as mean \pm standard deviation or $(\%)$; differences with a value of $\mathrm{P}<0.05$ were considered statistically significant. BMI: body mass index.

\section{Statistical analysis}

Data are presented as the mean \pm standard deviation (SD) for continuous variables or percentage for categorical variables. Quantitative data were compared between groups using Student's $t$-test or the Turkey-Kramer test. Differences in proportions were compared using Fisher's exact probability test. In the questionnaire on eating behaviors, the mean \pm SD was calculated for each item using dummy variables 1 - 4 described above. Nonparametric data were compared between groups using the Wilcoxon rank sum-test or Dunn's test. Analysis of variance was used to investigate the relations between BMI, SBP, fasting plasma glucose (FPG) in fifth grade, and the degree of habitual eating behaviors. Statistical analyses were performed using JMP software (version 15.0.0 for Macintosh; SAS Institute Inc., Cary, NC, USA). A value of $\mathrm{P}<0.05$ (two-sided) was considered significant.

\section{Results}

\section{Clinical characteristics and lifestyle profiles}

The participants' clinical characteristics and lifestyle profiles are shown in Table 1. Men had significantly greater height, weight, BMI, and SBP than women $(\mathrm{P}<0.001, \mathrm{P}<0.001, \mathrm{P}=$ 0.002 , and $\mathrm{P}<0.001$, respectively). No significant differences were seen in FPG, fasting plasma insulin, or weight change from first to fifth grade ( $\Delta$ weight) between men and women. In this study, regular exercise was identified in $48 \%$ of the participants, and the proportion was three times higher in men than in women ( $\mathrm{P}<0.001)$. The proportion of daily self-weighing was likely to be greater in women, whereas men were more likely to skip breakfast, engage in frequent late-night eating, and consume excessive amounts of soft drinks; however, none of the differences in lifestyle behaviors between men and women were significant.

\section{Comparison of clinical characteristics and lifestyle profiles according to daily behaviors}

Students were then divided into two groups according to lifestyle behaviors (daily self-weighing, regular exercise, skipping breakfast, frequent late-night eating, and excess soft drink consumption); their clinical characteristics and the proportion of daily behaviors are shown in Table 2 . No significant differences were found between groups according to self-weighing status. In addition, $83.3 \%$ of the students who reported engaging in regular exercise were man. BMI in both first- and fifth-grade students were significantly higher in the regular exercise group $(\mathrm{P}<0.05)$, and the percentage of students who consumed excessive amounts of soft drinks was three times higher in the regular exercise group $(\mathrm{P}<0.05)$. FPG was significantly higher in the skipping than in the not skipping breakfast group $(\mathrm{P}<0.005)$, and weight change from first to fifth grade ( $\Delta$ weight) tended to be greater in the skipping breakfast group $(\mathrm{P}=0.059)$. Age change after admission to university ( $\triangle$ age) was significantly greater in students who frequently ate late at night than in those 
Table 2. Comparison of Clinical Characteristics and Lifestyle Profiles Among University Students With or Without Daily Behaviors

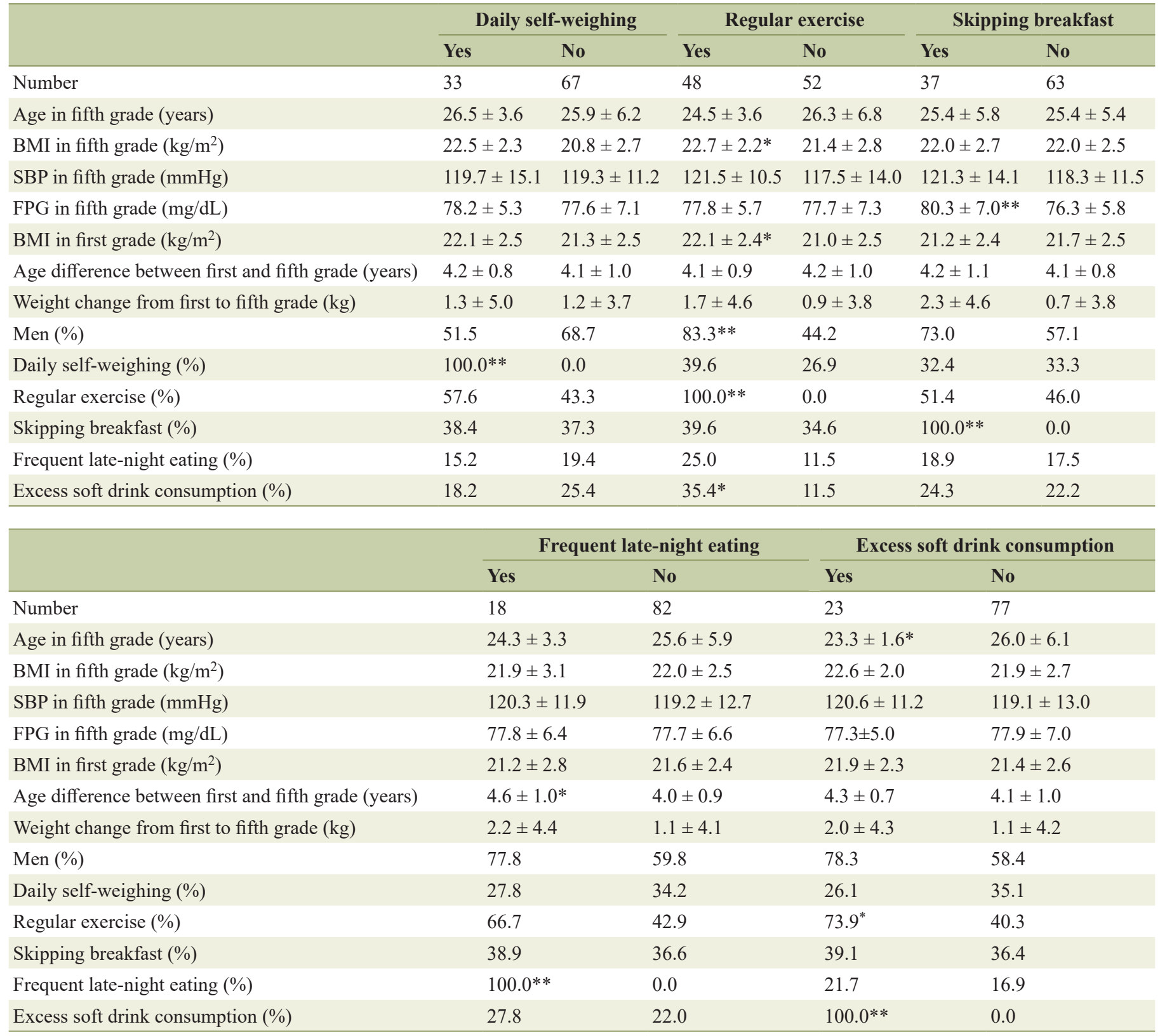

Data are expressed as mean \pm standard deviation or (\%). Statistically significant data; ${ }^{*} \mathrm{P}<0.05,{ }^{* *} \mathrm{P}<0.005$ vs. No. BMI: body mass index; SBP: systolic blood pressure; FPG: fasting plasma glucose.

who did not $(\mathrm{P}<0.05)$. The students who consumed excessive amounts of soft drinks were significantly younger and engaged in regular exercise significantly more frequently than those who did not $(\mathrm{P}<0.05$ and $\mathrm{P}<0.05$, respectively).

\section{Outcomes of the questionnaire on eating behaviors accord- ing to daily behaviors}

Comparing with and without lifestyle behaviors, the characteristics of eating behavior as quantified using dummy vari- ables are shown in Supplementary Material 1 (www.jofem. org). The results were as follows: 1) Students who weighed themselves daily believed that they were more likely to gain weight than those who did not. In addition, they often felt regretful after eating a lot, but refrained from ever eating fast food and sweet breads. 2) Students who engaged in regular exercise thought that it was more wasteful to leave food on a plate after a meal than those who did not, ordered great quantities of food, preferred noodles and meats, attended more banquets and drinking parties, considered dinner to be the most luxurious meal, and tended to eat until full. On the 
Table 3. Comparison of Clinical Characteristics, Lifestyle Profiles, and Responses on the Questionnaire on Eating Behaviors Based on the Difference in Weight Change From First to Fifth Grade

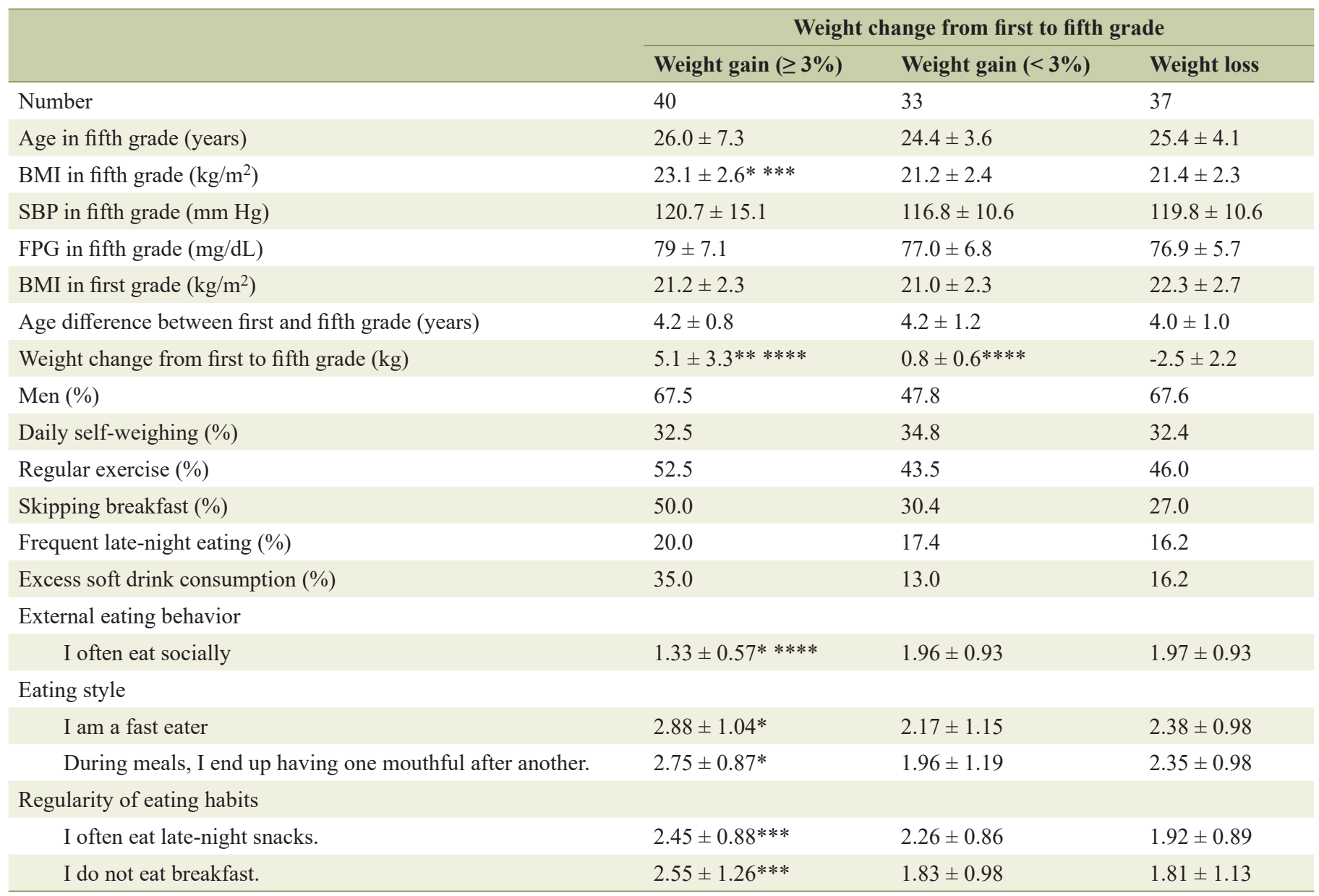

Data are expressed as mean \pm standard deviation or (\%). In the questionnaire on eating behaviors, only question items that had a significant difference are shown. Statistically significant data; ${ }^{*} \mathrm{P}<0.05$, ${ }^{* *} \mathrm{P}<0.005$ vs. weight gain $(<3 \%)$; ${ }^{* * *} \mathrm{P}<0.05$, ${ }^{* * * *} \mathrm{P}<0.005$ vs. weight loss. BMI: body mass index; SBP: systolic blood pressure; FPG: fasting plasma glucose.

other hand, students who did not engage in regular exercise were more likely to think that gaining weight was caused by a lack of exercise. 3) Students who skipped breakfast tended to be weaker in the morning than those who did not, to eat at irregular meal times, to not eat late-night snacks (but to eat dinner late), and to prefer fast food and meat over sweets. However, few of these students showed conditioned eating (e.g., "I eat because I don't like to waste food", "I eat because someone else is eating", "I eat because I am irritated", and "I eat even when I catch a cold"), and few indications were seen from others that they often ate well. 4) Students who frequently ate late at night often attended banquets or drinking parties, ate dinner late, kept food close and stocked food in the refrigerator, and tended to eat snacks, fruits, and sweets without thinking. However, they were not likely to think that they gained weight because of a lack of exercise. 5) Students who consumed excessive amounts of soft drinks had more irregular meal times than those who did not, preferred noodles and fast food, often ate out or got deliveries, and tended to eat a lot of snacks.
Comparison of clinical characteristics, lifestyle profiles, and responses to the questionnaire on eating behaviors based on the difference in weight change from first to fifth grade

The clinical characteristics, proportion of lifestyle behaviors, and questionnaire responses for eating behaviors for students grouped according to their change in weight are shown in Table 3 . BMI in fifth grade was significantly higher in the weight gain of $3 \%$ or more than in the weight gain of less than $3 \%$ group or the weight loss group $(\mathrm{P}<0.05$ and $\mathrm{P}<0.05$, respectively); however, no significant differences were seen between the weight gain of less than $3 \%$ and weight loss groups. In addition, no trends were observed for SBP or FPG. In the weight gain of $3 \%$ or more group, the percentage of students who consumed excessive amounts of soft drinks was twofold higher than that in the other groups; however, the proportions were not significantly different between the three groups. The students with weight gain of more than $3 \%$ had significantly greater self-awareness of fast eating, eating late-night snacks, 

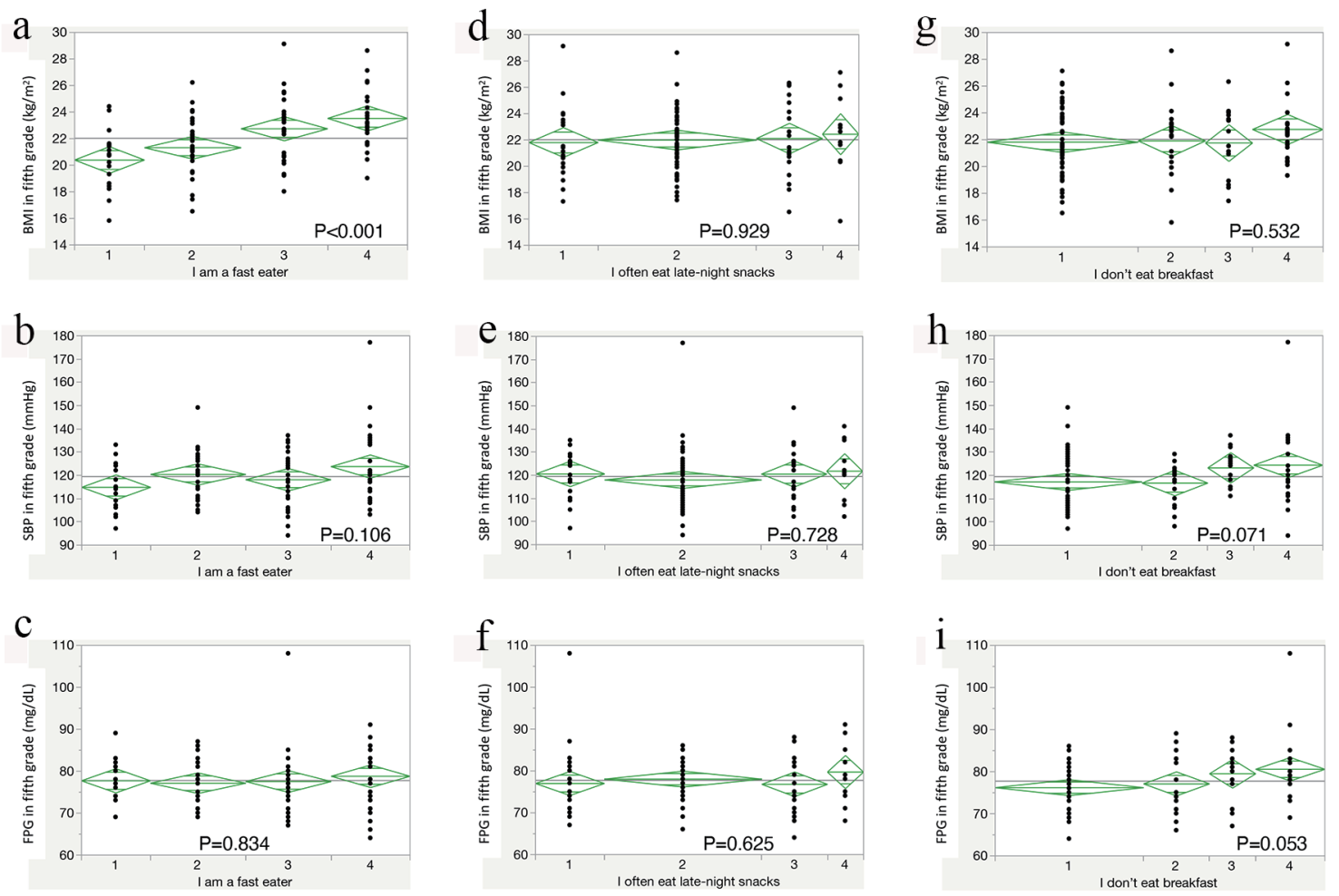

Figure 1. The relationship between BMI, SBP, and FPG in fifth grade and degree of self-awareness of habitual eating behaviors related to weight gain more than 3\%. (a-c) Fast eating; (d-f) Late-night eating; (g-i) Not eating breakfast. (a, d, g) BMI in fifth grade, (b, e, h) SBP in fifth grade, and (c, f, i) FPG in fifth grade. The numbers in each figure represent the degree of self-awareness of habitual eating behaviors: "1. Never, 2. Sometimes, 3. Frequently, and 4. Always". (a) The self-awareness of fast eating was significantly associated with higher BMI $(\mathrm{P}<0.001)$. (h, i) SBP and FPG tended to be higher in students who were aware of their tendency to skip breakfast. BMI: body mass index; SBP: systolic blood pressure; FPG: fasting blood glucose.

and not eating breakfast than those with weight gain of less than $3 \%$ or weight loss; however, they were also less aware that they would often eat while socializing.

\section{Relationships between BMI, SBP, and FPG in fifth grade and degree of self-awareness of habitual eating behaviors related to weight gain of more than $3 \%$}

Self-awareness of fast eating was significantly associated with higher BMI in fifth grade $(\mathrm{P}<0.001)$ (Fig. 1a); however, no significant relationship was found between SBP and FPG in fifth grade and the degree of self-awareness of fast eating (Fig. $1 \mathrm{~b}, \mathrm{c})$. Although no significant relationships were observed between BMI, SBP, and FPG in fifth grade and the degree of self-awareness of eating late at night or not eating breakfast (Fig. 1d-g), SBP and FPG in fifth grade tended to be higher in the students who were highly aware that they would not have breakfast ( $\mathrm{P}=0.071$ and $\mathrm{P}=0.053$, respectively) (Fig. 1h, i).

\section{Discussion}

In this study, based on the results of regular health checkups conducted at the time of university admission and in fifth grade and data obtained from clinical training, we examined the characteristics of eating behavior according to the backgrounds and lifestyles of university students and the factors involved in weight gain.

Although no difference in weight change was seen between the groups with or without daily weight measurement in this study, BMI was higher in the groups with a weight measurement habit. This could indicate that current weight per se is an important promoter of daily weight measurement (i.e., as persons gain weight, they start to measure their weight) [11]. The proportion of men was clearly higher in the groups engaging in regular exercise, and the physique and weight change patterns were actually similar between men and women (Table 1). Eating behavior in the group with exercise habits may be related to increased calorie consumption and is likely to reflect man characteristics. This is different from middle-aged and older people, in whom a lack of exercise habits leads to inadvertent weight gain [13]. It is also interesting that the students without exercise habits recognized that "they gain weight because of a lack of exercise". The students who skipped breakfast had few inadvertent eating behaviors, including conditioned eating; however, they also exhibited the greatest weight change pattern from first to fifth grade, which indicates that nocturnal life is apt to cause weight gain [6]. The students who frequently ate late at night took longer to progress from first to fifth grade, implying that they had to 
repeat courses. This could be the reason why they had many careless eating behaviors, such as keeping food around them and always eating between meals. While they were not likely to gain weight because of a lack of exercise, they firmly recognized that eating late-night snacks was a problem. The students who consumed excessive amounts of soft drinks were more likely to have exercise habits. Therefore, the overconsumption of soft drinks could be associated with exercise habits; this may be the reason why glucose tolerance was not significantly affected, as exercise habits compensate for the metabolic effects associated with excessive consumption of soft drinks [8]. When comparing the amount of weight change between the representative five lifestyles in this generation, eating habits generally had a stronger effect on weight change than did exercise habits (Table 2). Furthermore, it should be noted that the students who frequently ate late at night did not skip breakfast, and vice versa, and these two eating behaviors did not overlap with each other (Supplementary Material 1, www.jofem.org).

Regarding weight change, considering the weight itself in first grade and the influence of gender differences, we classified the students by the change rate, not by the change amount of the actual measurement value, and compared the weight gain of $3 \%$ or more, the weight gain of less than $3 \%$, and the weight loss groups. At our university, $37 \%$ of students lost weight after admission, but no difference in BMI in fifth grade was found between the weight loss and weight gain of less than 3\% groups (Table 3). No apparent difference in gender or lifestyle behaviors was observed, and BMI in first grade among the weight loss group was higher compared with the other two groups, which indicates that the students who were relatively heavy lost weight after entering university. However, since these two groups (weight loss and weight gain of less than 3\%) had primarily normal weight levels and were of average physique at the same age [14], weight gain of less than $3 \%$ can be interpreted as a natural gain for this generation. In fact, a significant difference in eating behaviors was observed in the weight gain of $3 \%$ or more group, and three unique eating behaviors ("fast eating", "eating late-night snacks", and "not eating breakfast") were extracted (Table 3). In multiple logistic regression analysis, "fast eating" and "not eating breakfast" were independent factors related to weight gain of $3 \%$ or more (data not shown). Furthermore, these two factors remained even if age, gender, various lifestyles, and BMI in first grade were used as confounding factors. Therefore, in this study, "fast eating" and "not eating breakfast" were independent factors for weight gain of $3 \%$ or more among this generation. In addition, "I often eat socially" was significantly low in the weight gain of $3 \%$ or more group, and the students who obviously gained weight overate on their own free will. When comparing the three eating behaviors in terms of self-awareness, BMI in fifth grade gradually increased depending on the cognitive strength of "fast eating" (Fig. 1a). The following mechanisms by which "fast eating" causes weight gain have been reported: due to a lack of satiety, "fast eating" may cause overeating before fullness, which can lead to insulin resistance, disrupted energy balance, and finally, overweight [15]. On the other hand, no differences in SBP, FPG (Fig. 1b, c), or fasting insulin level were seen (data not shown). Even in the group that was most aware of "fast eating", the average BMI was $23.5 \mathrm{~kg} / \mathrm{m}^{2}$, which is not considered obese, and insulin sensitivity was good; therefore, it may be difficult to observe metabolic changes compared with BMI in terms of self-awareness of "fast eating". On the other hand, high blood pressure and blood glucose levels tended to be observed in students who were strongly aware of "not eating breakfast" (Fig. $1 \mathrm{~h}, \mathrm{i})$. The students who skipped breakfast showed careful eating activities and a low desire for food; however, they led a nocturnal life and had trouble getting up in the morning, which indicates a high possibility of metabolic changes due to abnormal life rhythms rather than excessive energy intake. Skipping breakfast was most likely to cause weight change from first to fifth grade, but no difference was seen in BMI in fifth grade (Table 2). In this generation, nocturnal life rhythms tend to cause weight gain [6] and eating three proper meals a day has been reported to lead to obesity [16]. In other words, skipping breakfast tends to cause weight gain because of a shift to a nighttime lifestyle, but the absolute amount of energy intake is not necessarily large, so there may be no difference in BMI depending on the strength of awareness of "not eating breakfast". From the above, "fast eating" leads to weight gain and even obesity [17]. Even students who ate fast from time to time gained weight, so the BMI of students who develop a habit of "fast eating" should definitely be considered high. On the other hand, skipping breakfast and frequently eating at night also cause weight gain, but the current BMIs (in fifth grade) were not always higher than those of students who do not show this behavior (Table 2). However, if "not eating breakfast" becomes a habit, metabolic changes may occur easily regardless of the current weight. Being a member of society, experiencing an absolute decrease in regular exercise (e.g., withdrawing from club activities), and being exposed to gastronomy raise blood pressure and blood glucose levels. To correct fast eating, it is recommended to eat a good breakfast daily and practice chewing instruction. It is quite interesting that no differences were seen in the behaviors directly related to weight gain ("fast eating", "eating late-night snacks", and "not eating breakfast") with or without daily weight measurement, regular exercise, and excessive consumption of soft drinks (Supplementary Material 1, www.jofem.org).

In the questionnaire on eating behaviors, "I often eat latenight snacks" and "I don't eat breakfast" overlapped with the contents of the questionnaire items regarding the lifestyle behaviors "Frequently eating late at night" and "Skipping breakfast". Overall, 38.9\% of the students who replied "yes" to "Frequently eating late at night" answered "4. Always" for the item "I often eat late-night snacks" on the questionnaire on eating behaviors. Similarly, $56.8 \%$ of the students who replied "yes" to "Skipping breakfast" answered "4. Always" for the item "I don't eat breakfast". In other words, eating behavior varies from day to day, and self-assessment is difficult to evaluate using only dichotomous variables ("yes" or "no"), so this should be recognized as being fairly heterogeneous. By converting the results of the questionnaire on eating behaviors into a continuous scale, the students who answered "skipping breakfast" on the lifestyle-focused questionnaire were estimated to be twice as aware of "I don't eat breakfast" as those who did not, which suggests a large "perception gap" in the concerned eating behavior. On the other hand, "I often visit convenience stores" and "I like noodles" always had a score of 
more than 3 , regardless of lifestyle behaviors, and thus should be regarded as a general characteristic (habitual eating behavior) of this generation. In addition, the scores for "I am the type who gains weight even by drinking water" and "I can't tell if I'm hungry or full" were clearly low, which is specific to obese patients, and should be understood as a feeling close to an eating disorder. Although the questionnaire on eating behaviors was developed to treat obese patients, it also appears to be useful for understanding the habits and peculiarities of eating behaviors among university students.

The limitations of this study are as follows. First, this was a retrospective analysis of data obtained during clinical training, and the number of cases was limited to only 100 in one grade. Second, drinking and smoking history was excluded from the analysis because of the possibility of duplicate answers. Third, height, weight, and blood pressure data in fifth grade were obtained from regular health examinations in spring, and the blood sampling and questionnaire data were collected at the time of the clinical training. Accordingly, data should not be collected at the same time. Finally, living environment and stress have been reported to be factors that cause weight gain in university students $[3,18]$, so it is impossible to exclude the influence of these factors on eating behaviors.

In conclusion, we investigated the characteristics of eating behaviors based on the backgrounds and lifestyles of and the factors involved in weight gain for 100 fifth-grade students at Oita University Faculty of Medicine. The students who engaged in daily self-weighing took better care of their physique and constitution and avoided eating foods that cause weight gain. Regular exercise was common among men, and students with such habits tended to follow eating behaviors that compensated for the increase in calorie consumption. The students who skipped breakfast did not eat much, but their weight tended to increase due to their nighttime rhythm. The students who frequently ate at night often had snacks. The students who consumed excessive amounts of soft drinks often had careless eating behaviors but were also likely to have exercise habits. Three eating behaviors were extracted as factors that cause weight gain of 3\% or more: "fast eating", "eating late-night snacks", and "not eating breakfast". The habit of "fast eating" was found to cause weight gain, while the habit of "skipping breakfast" was shown to cause metabolic disorders easily regardless of current weight. These findings suggest that chewing instruction to correct fast eating and avoiding skipping breakfast to adjust students' daily rhythm should be part of health education for university students that aims to prevent metabolic syndrome.

\section{Supplementary Material}

Suppl 1. Outcome of questionnaire on eating behaviors in university students with or without daily behaviors.

\section{Acknowledgments}

We thank Keishi Mori for his assistance with the statistical analysis.

\section{Financial Disclosure}

None to declare.

\section{Conflict of Interest}

None to declare.

\section{Informed Consent}

Informed consent for blood sampling, collecting information concerning lifestyle behaviors, and responding to the questionnaire on eating behaviors was obtained from all students while in school. Because the students have already graduated, we announced that the opportunity to opt out was always available by providing relevant information on the website of our department. To date, there have been no such inquiries.

\section{Author Contributions}

TK contributed to conceptualization, methodology, software, formal analysis, investigation, writing-original draft and visualization. YY and MO contributed to investigation and data curation. HS was responsible for supervision and project administration. TT and YK contributed to writing (review and editing). All authors read and approved the final manuscript.

\section{Data Availability}

The authors declare that data supporting the findings of this study are available within the article.

\section{References}

1. Laitinen J, Power C, Jarvelin MR. Family social class, maternal body mass index, childhood body mass index, and age at menarche as predictors of adult obesity. Am J Clin Nutr. 2001;74(3):287-294.

2. Pliner P, Saunders T. Vulnerability to freshman weight gain as a function of dietary restraint and residence. Physiol Behav. 2008;93(1-2):76-82.

3. Kapinos KA, Yakusheva O. Environmental influences on young adult weight gain: evidence from a natural experiment. J Adolesc Health. 2011;48(1):52-58.

4. Nelson MC, Story M, Larson NI, Neumark-Sztainer D, Lytle LA. Emerging adulthood and college-aged youth: an overlooked age for weight-related behavior change. Obesity (Silver Spring). 2008;16(10):2205-2211.

5. Niemeier HM, Raynor HA, Lloyd-Richardson EE, Rogers ML, Wing RR. Fast food consumption and breakfast skipping: predictors of weight gain from adolescence to adulthood in a nationally representative sample. J Adolesc Health. 2006;39(6):842-849. 
6. Culnan E, Kloss JD, Grandner M. A prospective study of weight gain associated with chronotype among college freshmen. Chronobiol Int. 2013;30(5):682-690.

7. Bawadi H, Khataybeh T, Obeidat B, Kerkadi A, Tayyem R, Banks AD, Subih H. Sugar-sweetened beverages contribute significantly to college student's daily caloric intake in Jordan: soft drinks are not the major contributor. Nutrients. 2019;11(5):1058.

8. Kakuma T, Ariki S, Yoshida Y, Sato A, Shibata H, Tsutsumi T, Kudo Y. The examination about glucose metabolism using 75-g oral glucose tolerance tests in fifth-grade students at Oita University, Faculty of Medicine; the first report. Campus Health. 2019;56(2):82-88. Japanese.

9. Obesity care guideline development committee. In: Obesity care guideline 2016. Tokyo: Life science. 2016; p. 40-43. Japanese.

10. Willett WC, Sampson L, Stampfer MJ, Rosner B, Bain C, Witschi J, Hennekens $\mathrm{CH}$, et al. Reproducibility and validity of a semiquantitative food frequency questionnaire. Am J Epidemiol. 1985;122(1):51-65.

11. Kakuma T, Ariki S, Yoshida Y, Shibata H, Tsutsumi T, Kudo Y. Relation between daily self-weighing and physique, lifestyle factors, and glycemic parameters in Japanese college students. J Endocrinol Metab. 2020;10:8-15.

12. Anonymous. Metabolic syndrome definition and diagnos- tic criteria in Japan. J Jpn Soc Int Med. 2005;94:794-809. Japanese.

13. Jakicic JM, Powell KE, Campbell WW, Dipietro L, Pate RR, Pescatello LS, Collins KA, et al. Physical activity and the prevention of weight gain in adults: a systematic review. Med Sci Sports Exerc. 2019;51(6):1262-1269.

14. Japanese National University Council of Health Administration Facilities. White paper: University Student Health 2015. Nagoya: Nagoya University Co-op; 2018. Japanese.

15. Sun Y, Sekine M, Kagamimori S. Lifestyle and overweight among Japanese adolescents: the Toyama Birth Cohort Study. J Epidemiol. 2009;19(6):303-310.

16. Ibe Y, Miyakawa H, Fuse-Nagase Y, Hirose AS, Hirasawa R, Yachi Y, Fujihara K, et al. Association of eating three meals irregularly with changes in BMI and weight among young Japanese men and women: A 2-year follow-up. Physiol Behav. 2016;163:81-87.

17. Yamane M, Ekuni D, Mizutani S, Kataoka K, SakumotoKataoka M, Kawabata Y, Omori C, et al. Relationships between eating quickly and weight gain in Japanese university students: a longitudinal study. Obesity (Silver Spring). 2014;22(10):2262-2266.

18. Pelletier JE, Lytle LA, Laska MN. Stress, health risk behaviors, and weight status among community college students. Health Educ Behav. 2016;43(2):139-144. 